\title{
teoría del efecto de vibraciones producidas por choques o explosiones sobre estructuras e instalaciones
}

MARIANO FERNANDEZ BOLLO

Director Técnico de la Sociedad de Reconocimientos Geofísicos, S. A.

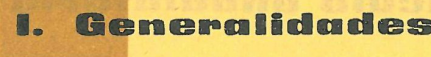

Las vibraciones $\mathrm{u}$ oscilaciones producidas por sismos, explosiones o choques violentos producen averías, desperfectos y, a veces, la ruina total de estructuras e instalaciones.

El estudio de los efectos sísmicos o de terremotos, por su extensión e importancia, ha sido objeto de muchas investigaciones importantes y forma una parte completa de la técnica de proyectar.

Actualmente, tiene también gran importancia la previsión de los efectos que pueden producir explosiones y choques, fenómenos físicos cuya intensidad y frecuencia crece con el progreso de la técnica. Una literatura técnica bastante extensa existe sobre el tema, de la cual se citan algunos de los trabajos más relacionados con estas notas al final de las mismas.

Las consecuencias de las vibraciones pueden variar entre las averías graves o ruina de las estructuras o instalaciones y la simple producción de averías o pequeños desperfectos.

Es necesario unir siempre al concepto de la avería el de su probabilidad, ya que la experiencia indica que los procesos que se producen por causa de las vibraciones son tan complejos que el resultado final puede ser muy variable.

Así, un cierto nivel del fenómeno diremos que tiene riesgo de averías ligeras o de averías graves. La definición de este límite de riesgo puede servir también de base para el estudio de seguros, responsabilidades e indemnizaciones, así como para evaluar el gasto razonable en precauciones y elementos de seguridad suplementaria.

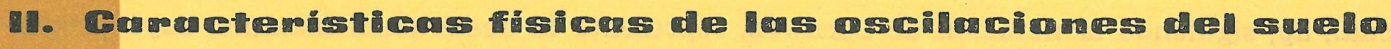

En las oscilaciones del terreno intervienen las variables ligadas a la estructura geológica del mismo y a las características físicas de cada una de las formaciones que constituyen esta estruc.tura. Además, es preciso tener en cuenta en el análisis del fenómeno las distintas ondas que pueden presentarse, que tienen distintas características en tanto que cscilación propiamente dicha.

Las caracteristicas fisicas de las formaciones geológicas que influyen, principalmente, en las oscilaciones y en su efecto sobre las estructuras son:

Módulo elástico $=\mathrm{E}$.

Coeficiente de Poisson $=\sigma$.

Coeficiente de viscosidad elástica $=\pi$.

La forma geométrica y condiciones en los limites (superficie libre, unión más o menos rígida con otro material, presión). 


\section{Perturbaciones transitorias}

Las perturbaciones transitorias no suelen tener importancia más que en los casos de oscilaciones continuas, como en fábricas y centrales en los casos de choque aislado o explosión.

En la práctica, las magnitudes esenciales son la frecuencia y la aceleración máxima. La frecuencia condiciona los fenómenos de resonancia, que son normalmente los más graves, y la aceleración máxima se relaciona con los de fatiga del material y eventual rotura.

A falta de información más completa, se consideran como límites normales admisibles para la frecuencia máxima $35 \mathrm{hz}$. Para la aceleración máxima bajo este valor se suele admitir 0,001 $\mathrm{g}$ en zonas tranquilas y $0,005 \mathrm{~g}$ en zonas ruidosas. En frecuencias superiores a $35 \mathrm{hz}$ sólo es posible admitir de $1 / 5$ a $1 / 20$ de estas cifras.

Estos datos son puramente indicativos y exigen en cada caso un estudio del problema y de las características de resonancia de los elementos que pueden sufrir perturbaciones. Para los seres humanos, las más incómodas son las de 1.000 a $2.000 \mathrm{hz}$ y las de $0,5 \mathrm{a} 4 \mathrm{hz}$ habitualmente.

\section{Perturbaciones que pueden causar averias}

Las averías aparecen por superposición de las tensiones propias del elemento averiado con las producidas por la oscilación.

Existen tres tipos o intensidades diferentes de perturbación:

Ondas de rotura muy intensas, que son capaces de producir por sus propios esfuerzos la rotura del material.

Ondas de intensidad suficiente para producir averias en los elementos de estructuras o instalaciones, por acción de unos elementos sobre otros.

Ondas débiles que pueden producir averías por acumulación de energía en fenómenos de resonancia.

Las primeras ondas son las que producen la rotura de la roca o de las fábricas en casos de excavación con explosivos; intensos terremotos y voladuras. Las del tipo segundo son las que normalmente producen las averías o ruinas debidas a terremotos o explosiones importantes. Se produce la rotura de los soportes de las masas importantes sometidas a oscilaciones, con poca impedancia sísmica y débil amortiguamiento.

El tipo tercero produce averías por fatiga o resonancia aguda y es el más corriente en choques débiles repetidos, que vienen a constituir una transición entre las oscilaciones continuas y las aisladas. Es el caso de las vías con intensa circulación, la proximidad de trabajos con maquinaria pesada y otros análogos.

\section{Limites peligrosos al primer tipo de perturbación}

La teoría de Hino (op. cit.), permite estudiar matemáticamente el proceso de rotura con bastante coincidencia con los resultados prácticos. Introduce el concepto de indice de susceptibilidad a la voladura:

$$
\mathbf{B}=\frac{\mathbf{S}_{\mathbf{c}}}{\mathbf{S}_{\mathbf{t}}}
$$

en que $S_{c}$ y $S_{t}$ representan las resistencias a la compresión y a la tracción del medio.

Para que se produzca la rotura es preciso que la tensión máxima producida por la oscilación sobrepase la resistencia del medio, la menor de las cuales es $\mathbf{S}_{t}$, y la máxima compresión admisible en la onda producida por el fenómeno es $\mathbf{S}_{\text {c }}$. Resulta de aquí que las oscilaciones peligrosas aparecen desde que las tensiones máximas alcanzan el valor $\mathbf{S}_{\text {c }}$.

Se puede establecer así un límite teórico:

$$
\mathrm{A} \cdot f=\mathrm{K} \frac{\mathbf{S}_{\mathrm{t}}}{\delta x}
$$


que se puede escribir también

$$
a g=4 \pi^{2} \mathrm{~K} \frac{\mathrm{S}_{\mathrm{t}}}{\delta} \cdot \frac{f}{\alpha}
$$

donde $\mathrm{K}$ es un coeficiente de distribución de tensiones instantáneas.

$\frac{f}{\alpha}$ de $5 \cdot 10^{-5}$ (unidades c.g.s.) define valores de $a$ del orden de 1 a 10 .

El coeficiente de susceptibilidad B de estos materiales varía de 80 a 10 como orden de magnitud para $\mathbf{S}_{\mathrm{c}}>30 \mathrm{~kg} / \mathrm{cm}^{2}$.

\section{Limites peligrosos al segundo tipo de perturbación}

La producción de esfuerzos por oscilación de elementos de las estructuras o instalaciones es difícil de estudiar de una manera general, ya que sus posibilidades de oscilación son sumamente variables.

En la práctica se adoptan, generalmente, dos criterios de limitación de riesgo:

El límite del valor de $a$ (aceleración máxima).

La máxima energía relativa $q$ admisible.

El criterio fundado en el valor de $a$ se ajusta mejor a las observaciones medias reales en edificios y estructuras para frecuencias inferiores a $15 \mathrm{hz}$ y el basado en $q$ para instalaciones, estructuras metálicas medias y edificios metálicos para frecuencias superiores a $5 \mathrm{hz}$.

Los valores límites admisibles de $a$ y $q$ más generalmente utilizados varían para $a$ de 0,05 a 0,25 y para $q$ de 0,20 a 0,50 .

Los valores $a=0,1$ y $q=0,28$ se han utilizado con frecuencia como límite práctico admisible para la presencia de un riesgo de averías poco importantes.

\section{El riesgo de perturbaciones del tercer tipo}

Es más difícil de evaluar en forma general que en los casos anteriores. La magnitud más importante para estudiar el riesgo de resonancia es la frecuencia $f$.

El único procedimiento riguroso que permite la investigación de estos fenómenos es la determinación de las frecuencias de los elementos componentes de la instalación y de la perturbación.

Cuando no se dispone de estos datos y desde un punto de vista general se puede admitir, de acuerdo con la experiencia límite basado en la energía relativa $q$. El riesgo de resonancias se puede graduar en tres tipos:

Riesgo de resonancia en elementos poco sensibles (ventanas, mobiliario) y del orden de $10^{-3}$ o mayor.

Riesgo sólo en elementos sensibles del orden de $10^{-5}$ o mayor.

No existe prácticamente riesgo para $q$ del orden de $10^{-7}$ o menor, salvo en máquinas 0 aparatos excepcionales.

Desde el punto de vista de la frecuencia, no es posible fijar con exactitud reglas generales, pero como orientación puede estimarse que presentan mayor riesgo las inferiores a $20 \mathrm{hz}$ respecto a la resonancia de conjuntos y las superiores a $2.000 \mathrm{hz}$ respecto a la resonancia de elementos o piezas aisladas de elevada rigidez. 


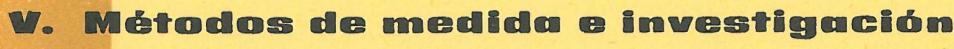

Sólo consideramos esta cuestión desde un punto de vista general, con el fin de permitir la aplicación de los datos y fórmulas anteriores a los casos prácticos.

\section{Fórmulas generales empiricas}

La experiencia de la utilización de explosivos ha permitido la determinación de fórmulas generales que pueden orientar acerca del orden de magnitud de las oscilaciones o vibraciones que producirá un tiro. Nuestros estudios y la experiencia de unos 150 ensayos nos han permitido establecer también una fórmula aproximada de orientación para un choque sobre un macizo de hormigón.

Estas fórmulas no pueden reemplazar, sin embargo, a las garantías más completas que da una investigación experimental real.

Thoenen y Windes, citados también por Cornwell (op. cit.), establecieron una fórmula consecuencia de numerosos tiros de voladura en cantera de roca consistente que define la amplitud:

$$
\mathrm{A}=\mathrm{N} \cdot 0,03 \mathrm{Q}^{2 / 3}\left(e^{-0,0047 \mathrm{r}}+0,0143\right)
$$

donde $\mathbf{Q}$ es la carga de dinamita en $\mathrm{kg}, \mathrm{y} r$ indica la distancia en metros.

Los valores de amplitud que da esta fórmula se multiplicaron por un factor $\mathrm{N}$ variable entre 0,1 y 3,0 , según se trate de tiros al aire o bajo una gran cobertura de roca, tal como $50 \mathrm{~m}$ de roca en estratos o capas gruesas.

La apreciación o previsión de la frecuencia se puede realizar por la siguiente escala.

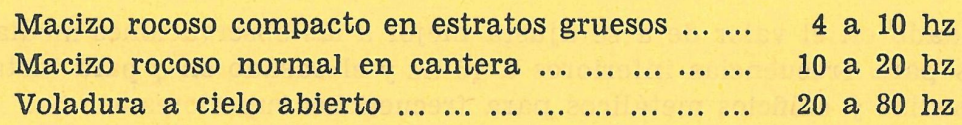

Recomiendan sus autores el empleo de la frecuencia $10 \mathrm{hz}$ para la previsión del riesgo de inmuebles de vivienda, ya que parece dar resultados prudentes.

Los límites prácticos de validez de la fórmula son:

\section{CARGAS}

$\begin{array}{rrrr}5 & \text { a } & 50 & \mathrm{~kg} \\ 50 & \mathrm{a} & 500 \mathrm{~kg} \\ 500 & \text { a } & 7.500 & \mathrm{~kg}\end{array}$

\section{DISTANGIAS}

$30-300 \mathrm{~m}$

$30-1.800 \mathrm{~m}$

$150-1.800 \mathrm{~m}$

Para muchos tiros usuales esta fórmula no es aplicable por corresponder a cargas fuertes y distancias grandes.

Crandell (op. cit.) y Cornwell (op. cit.) aconsejan la fórmula debida al primero de estos autores:

$$
q=1,1 \cdot P \cdot\left(\frac{Q}{r}\right)^{2}
$$

donde $\mathrm{P}$ varía de 1 a 4 al pasar de la roca consistente a la arcilla. Cornwell aconseja combinar las fórmulas [19] y [20], cuando ello es posible, para obtener límites más próximos a los que daría la experimentación real.

La validez de [20] se encuentra limitada por cargas de 05 a $50 \mathrm{~kg}$ y distancias de 7,5 a $75 \mathrm{~m}$.

Si bien estas fórmulas no pueden reemplazar una medida experimental real, evitarán graves errores y pueden dar idea del interés o no de la experimentación.

En los choques sobre hormigón, basándose en los resultados de series de experiencias en San Esteban (Galicia), Meyrargues (Provenza), St. Michel (Bretaña), Mallemort (Provenza) y Carla (Macizo Central), se puede establecer:

$$
q=\mathrm{P}\left(\frac{\mathrm{M} \cdot h}{r}\right)^{2}
$$


donde $\mathrm{M}$ es una masa de 2 a $20 \mathrm{~kg}$ de acero; $h$ representa la altura de caída sobre una superficie horizontal (2 a $4 \mathrm{~m}$ ), y $r$ expresa la distancia en metros de 1 a 10.

El coeficiente $P$ varía de unos a otros ensayos entre 0,003 y 0,005 , salvo en el caso de que el bloque de hormigón sea una placa de 0,40 a $0,20 \mathrm{~m}$ de espesor, en que llega a 0,02 para $10 \mathrm{~kg}$ de peso y $2 \mathrm{~m}$ de altura.

Esta fórmula puede ser útil para el desplazamiento de máquinas si se utiliza su extrapolación simplemente a título orientativo.

\section{Equipos y medios de investigación experimental directa}

Para determinar las características de las oscilaciones reales y poder efectuar ensayos que presenten suficiente garantía contra el riesgo sin limitar excesivamente la utilización de los agentes perturbadores es preciso utilizar equipos y aparatos de medida de las características citadas en el capítulo II y que intervienen en las fórmulas.

También existen dispositivos de control y alerta para indicar que las vibraciones se aproximan a niveles peligrosos en una zona.

1

PUNTO DE EXPLOSION<smiles>C1=C[As]1</smiles><smiles>[C+]1[CH]CCC1</smiles>

\section{Posición general}

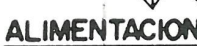

\section{a) Aparatos \\ $y$ disposirivos \\ de investigación}

Los aparatos y dispositivos de estudio de oscilaciones se agrupan en dos tipos generalmente designados como sismómetros y sismógrafos, respectivamente.

Los sismómetros son aparatos que determinan alguna característica de la oscilación, tal como la celeridad, la energía relativa, la aceleración máxima, la amplitud o la frecuencia. De ahí que en cada caso se designen también como frecuencímetros, acelerómetros, etc.

Los sismógrafos son equipo que registran las oscilaciones para permitir su estudio. Pueder ser de tipos muy diversos y frecuentemente se trata de aparatos de varios "canales", que permiten el registro simultáneo de varias vibraciones u oscilaciones. En ellos se pueden distinguir tres elementos o aparatos en cada canal, con misiones bien diferenciadas: el captor que transforma la oscilación en magnitudes eléctricas, el amplificador-transductor, que permite reforzar la señal inicial, transformándola en indicación gráfica, y el registrador propiamente dicho, que suministra el documento gráfico correspondiente. 
En todos los casos las indicaciones obtenidas deben ser investigadas teniendo en cuenta el tipo de aparato de ensayo empleado. Los sismógrafos de tipo "inductivo" transmiten las celeridades de las oscilaciones; los "acelerómetros", como su nombre indica, las aceleraciones. Se pueden construir captores de tipo "absoluto", basados generalmente en una variación de capacidad, que transmiten los desplazamientos reales del punto estudiado.

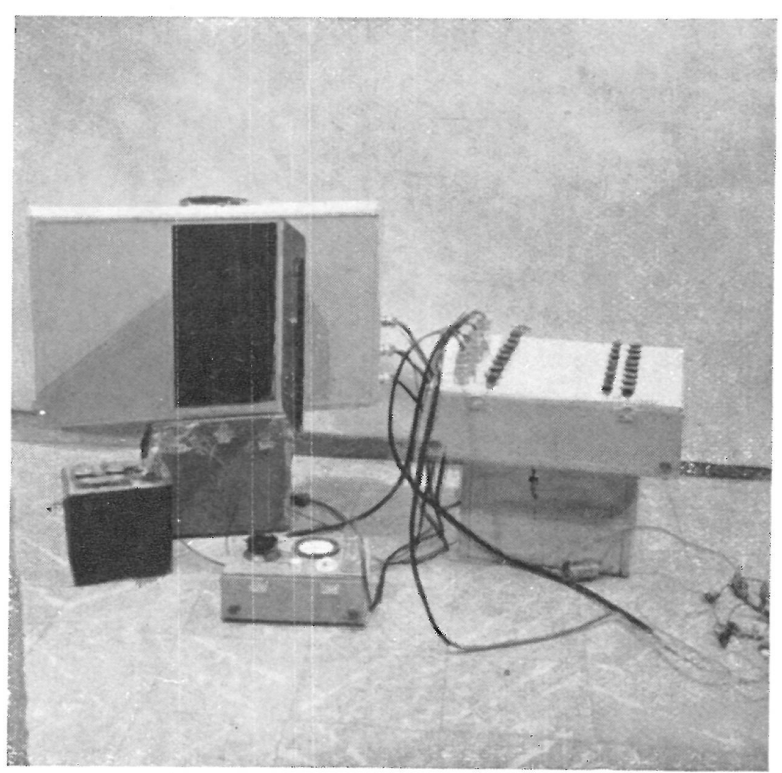

2

El registrador debe ser adecuado para las frecuencias a estudiar, con una curva de respuesta válida. Para ello es preciso evitar, por encima de 200 a $1.000 \mathrm{hz}$ los órganos móviles y utilizar los tubos de rayos catódicos o equivalentes.

En las figuras adjuntas damos una idea de varios de los equipos que se utilizan para estas investigaciones.

\section{b) Dispositivos de control}

Entre los dispositivos más interesantes y sencillos para esta aplicación se encuentran los "acelerómetros" descritos por Langsford (op. cit.), constituídos por una serie de varillas de acero de 6,35 milímetros de diámetro y longitudes escalonadas de 152 a $381 \mathrm{~mm}$.

Las oscilaciones cuya aceleración instantánea es suficientemente elevada y con una frecuencia adecuada originan su vuelco" El simple examen después del tiro o choque indica si se ha sobrepasado o no el límite correspondiente. Un estudio cuidadoso de su funcionamiento (véase Bollo: op. cit.), señala que para obtener con este dispositivo indicaciones seguras es preciso disponer varias series de varillas y operar con resultados medios.

Otro tipo de acelerómetro más compacto, pero menos utilizado, es el de esferas de acero con vuelta automática y registro de sus desplazamientos. Como se puede observar en la figura adjunta, el contador eléctrico automático permite apreciar. si los límites de oscilación fueron sobrepasados.

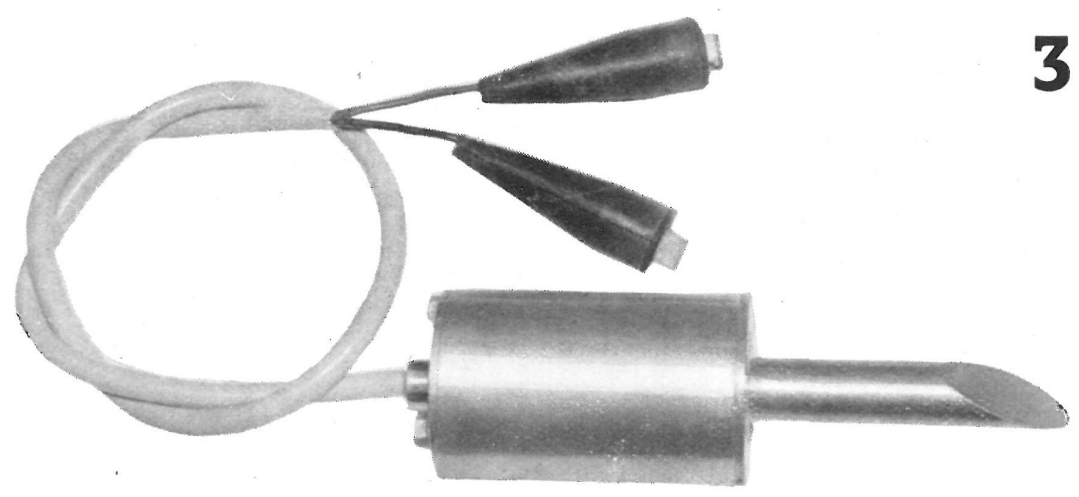

El empleo de estos dispositivos de una forma racional debe ser consecuencia de un estudio experimental de las vibraciones, lo cual permite la definición de los límites de riesgo en cada caso. Si esto no fuese posible se pueden utilizar las características de fabricación de los equipos para casos usuales y tomar un margen de seguridad sobre el valor de la energía relativa $q$ para estos casos delicados. Es importante tener en cuenta que en este tipo de fenómenos un escalón de seguridad tipo es la modificación de $q$ siguiendo el factor 10. Es decir, que modificaciones razonables serán reducir $q$ a 1/10 ó 1/100 del límite general, según el caso planteado. 


\section{b K $\delta$}

3/O N3 SHIN3กJ3Y]

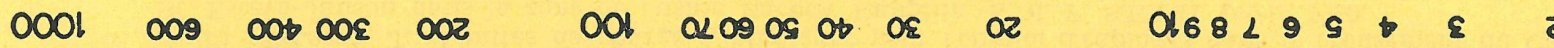

Axin

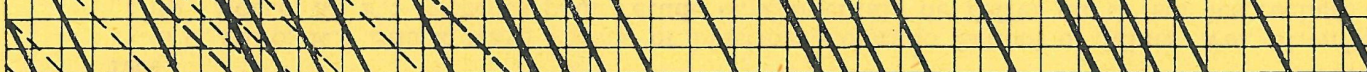

N N W $200^{\circ}$

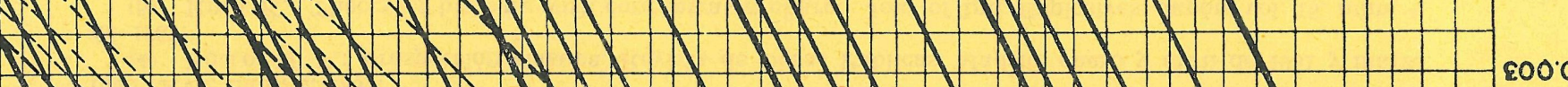

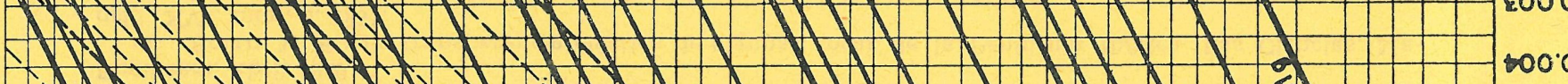

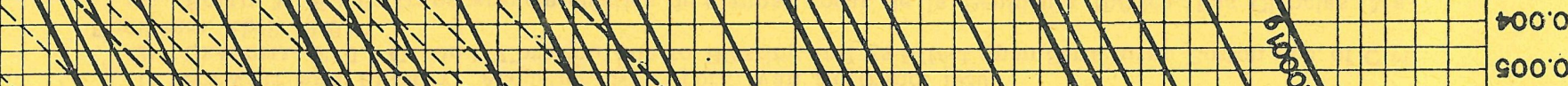

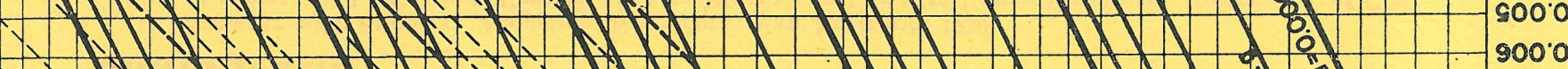

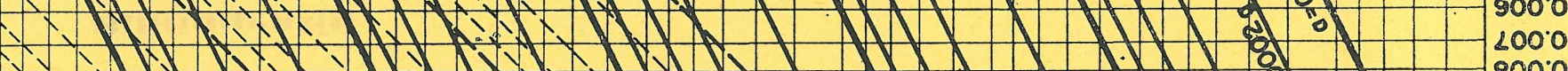

江

N.

(1)

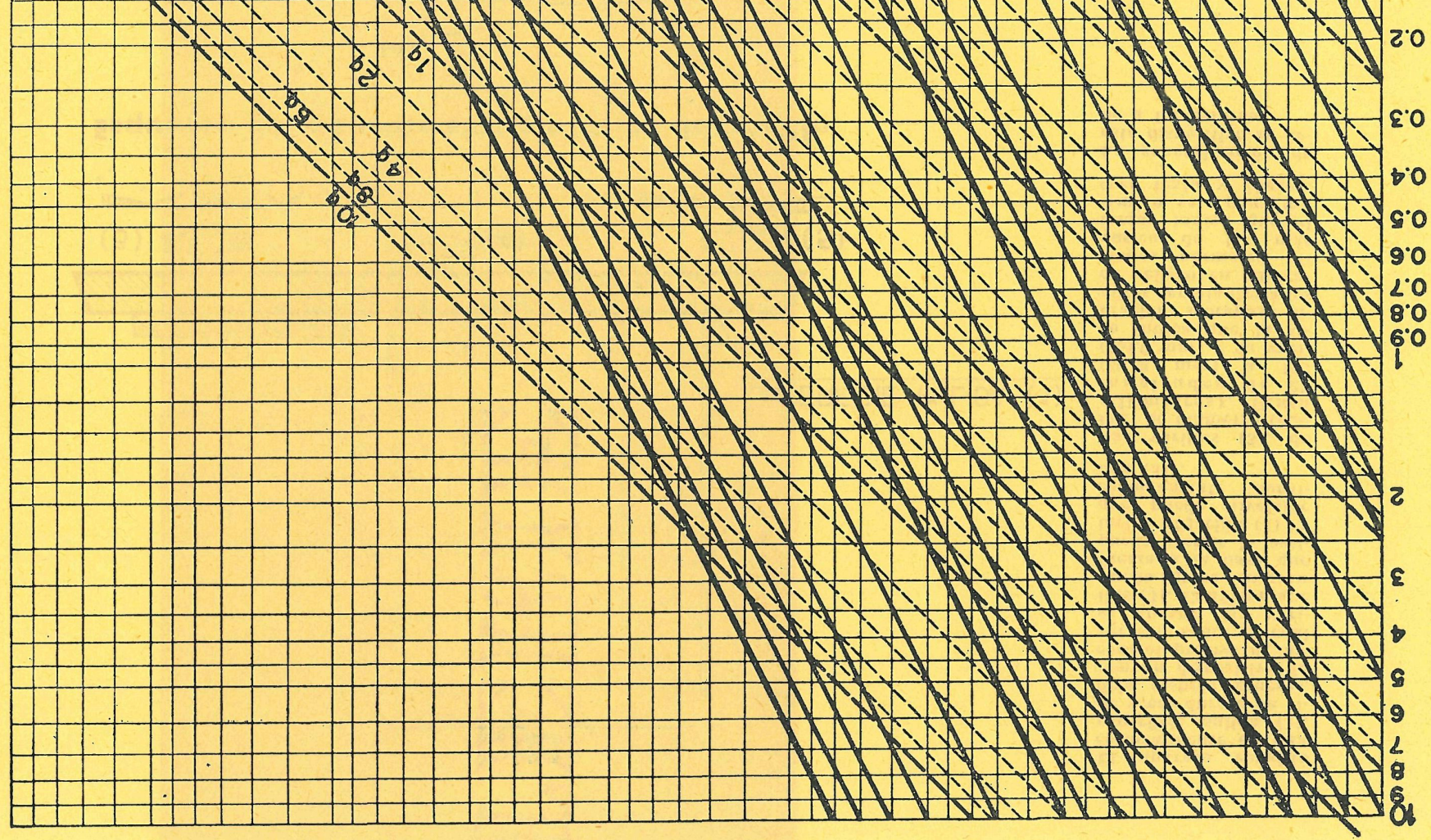



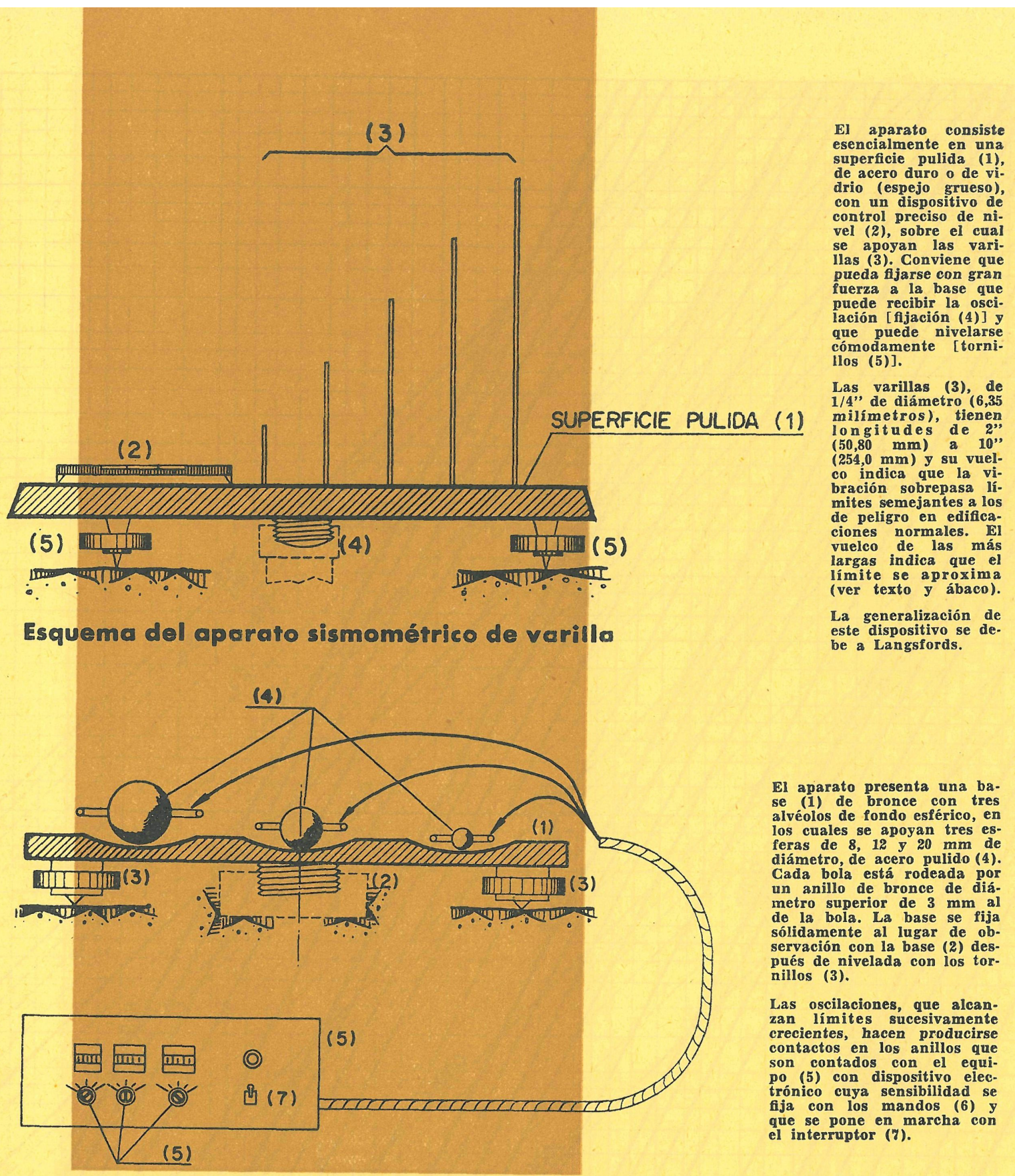

El aparato presenta una base (1) de bronce con tres alvéolos de fondo esferico, en los cuales se apoyan tres esferas de 8, 12 y 20 min de Cada bola esté rodeada por un anillo de bronce de diámetro superior de $3 \mathrm{~mm}$ al de la bola. La base se fija sólidamente al lugar de observación con la base (2) después de nivelada con los tornillos (3).

Las oscilaciones, que alcan. zan limites sucesivamente crecientes, hacen producirse contactos en los anillos que son contados con el equipo (5) con dispositivo electrónico cuya sensibilidad se fija con los mandos (6) y el interruptor $(y)$.

\section{Esquema del aparato sismométrico de esferas}

\section{Bibliog ro caf fía}

(1) StrWART and LINDSAy: Acoustics. New York. Van Nostrand, 1930.

(2) Crandeli, F. J.: Ground Vibration due to Blasting and its Effect upon Structures. Boston Soc. of Civil Engineers. Abril 1949.

(3) Bollo, M. F.: La transparencia elástica de algunas rocas de la Península Tbérica. Las Ciencias. Madrid, XVI, núm. 3, 1951.

(4) Bollo, M. F.: Prospección elástica. Revista de Obras Públicas. Madrid, enero y abril de 1951 y marzo de 1952 .

(5) HnNo, K.: Theory of blasting with concentrated charge. Jour of Ind. Explosives. Japón, vol. 15, núm. 4, 1954.

(5') Hrvo, K.: Shock wave theory of blasting. Colorado School of Mines. Vol. 51, núm. 3, 1956.

(6) Bollo, M. F.: Procédés pour l'étude expérimentale de l'effet du tir sur les roches. Journées de Méc. des Roches, París, Juin de 1957.

(7) CoRnWell, F. E.: Les effects des ébranlements causés par les tirs de mine à proximeté d'edifices ou d’ouvrages. Manuel du Creusement du Rocher. A.B. Atlas Diesel, Stockholm, 1958.

(8) TALOBRE, J.: Limites de charge à appliquer pour l'emploi d'explosifs dans le creusement du Canal de St. Esteve-Janson dans la zone de l'usine Barbier Dauphin. E. d. F. S.I.P.H. París, 1959.

(9) Bollo, M. F.: Methode sismo-élastique dans les recherches géomécaniques. Laboratorio Nacional de Engenharia, Lisboa, Outubro, 1960.

(10) Bollo, M. F.: Exploitation de la carrière Beck à Mallemort. S.R.G. París, 1961. 